\title{
Wilson Fermions, Random Matrix Theory and the Aoki Phase
}

\author{
Gernot Akemann \\ Department of Mathematical Sciences \& BURSt Research Center \\ Brunel University West London, Uxbridge UB8 3PH, United Kingdom \\ E-mail: gernot .akemann@brunel .ac.uk
}

\section{Poul H. Damgaard}

The Niels Bohr International Academy and Discovery Center

The Niels Bohr Institute, Blegdamsvej 17, DK-2100 Copenhagen, Denmark

E-mail: phdamg@nbi.dk

\section{Kim Splittorff}

The Niels Bohr Institute, Blegdamsvej 17, DK-2100 Copenhagen, Denmark

E-mail: split@nbi.dk

\section{Jacobus Verbaarschot*}

State University of New York

Department of Physics and Astronomy

Stony Brook, NY 11794-3800, USA

E-mail: jacobus.verbaarschotestonybrook.edu

\begin{abstract}
The QCD partition function for the Wilson Dirac operator, $D_{W}$, at nonzero lattice spacing $a$ can be expressed in terms of a chiral Lagrangian as a systematic expansion in the quark mass, the momentum and $a^{2}$. Starting from this chiral Lagrangian we obtain an analytical expression for the spectral density of $\gamma_{5}\left(D_{W}+m\right)$ in the microscopic domain. It is shown that the $\gamma_{5}$-Hermiticity of the Dirac operator necessarily leads to a coefficient of the $a^{2}$ term that is consistent with the existence of an Aoki phase. The transition to the Aoki phase is explained, and the interplay of the index of $D_{W}$ and nonzero $a$ is discussed. We formulate a random matrix theory for the Wilson Dirac operator with index $v$ (which, in the continuum limit, becomes equal to the topological charge of gauge field configurations). It is shown by an explicit calculation that this random matrix theory reproduces the $a^{2}$-dependence of the chiral Lagrangian in the microscopic domain, and that the sign of the $a^{2}$-term is directly related to the $\gamma_{5}$-Hermiticity of $D_{W}$.
\end{abstract}

The XXVIII International Symposium on Lattice Field Theory, Lattice2010

June 14-19, 2010

Villasimius, Italy

\footnotetext{
${ }^{*}$ Speaker.
} 


\section{Introduction}

For sufficiently small eigenvalues the spectrum of the QCD Dirac operator can be obtained from the zero momentum part of a chiral Lagrangian that is consistent with the global symmetries of QCD [1]. The reason is spontaneous symmetry breaking and the existence of a mass gap, so that QCD at low energy is described by a chiral Lagrangian for the Goldstone bosons. If the Goldstone bosons corresponding to the mass scale of the Dirac spectrum we are interested in, have a Compton wavelength that is much large than the size of the box, the zero momentum part of the partition function, which determines its mass dependence, factorizes from the nonzero momentum part [2]. All theories with a mass gap and the global symmetries and symmetry breaking pattern of QCD are described by the same zero momentum chiral Lagrangian. Analyzing the simplest such theory, chiral random matrix theory, allows us to answer questions that cannot be addressed otherwise.

The Dirac operator in lattice QCD has discretization errors, and if the discretization errors change the underlying symmetries, the low lying Dirac spectrum will be affected significantly. This is the case for the Wilson Dirac operator $D=D_{W}+m$, where

$$
D_{W}=\frac{1}{2} \gamma_{\mu}\left(\nabla_{\mu}+\nabla_{\mu}^{*}\right)-\frac{1}{2} a \nabla_{\mu}^{*} \nabla_{\mu}
$$

is written in terms of forward $\left(\nabla_{\mu}\right)$ and backward $\left(\nabla_{\mu}^{*}\right)$ covariant derivatives, and $m$ is the quark mass. The Wilson term violates the axial symmetry but the Dirac operator remains $\gamma_{5}$ Hermitian

$$
D_{W}^{\dagger}=\gamma_{5} D_{W} \gamma_{5}
$$

Because of this, the operator $D_{5} \equiv \gamma_{5}\left(D_{W}+m\right)$ is Hermitian, and is computationally much simpler to work with. Its spectrum has been analyzed in lattice QCD simulations $[4,5]$ and by means of mean field studies of chiral Lagrangians [6,7].

For $a=0$, the spectrum of $D_{5}$ has a gap $[-m, m]$. At nonzero $a$, states intrude inside the gap, and for sufficiency large $a$, the gap closes, and we enter what is known as the Aoki phase (see Fig. 1). In terms of $D_{W}$, this is the point where $m$ hits the cloud of eigenvalues (see Fig. 1, right).
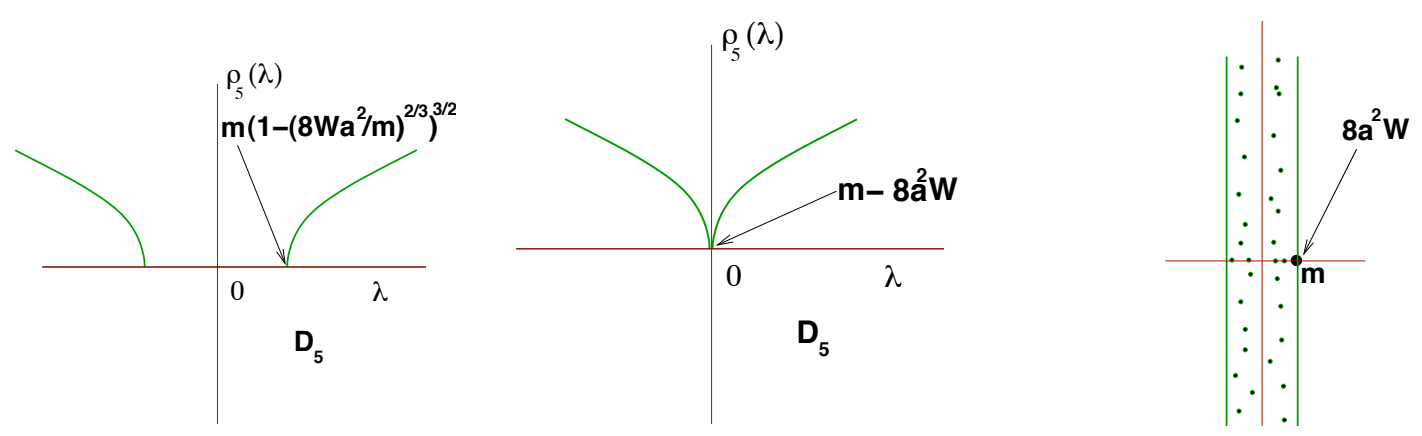

Figure 1: Schematic picture of mean field results for the spectral density of $D_{5}$ (left two figures) and the spectrum of $D_{W}$ (left). The right two figures show the onset of the Aoki phase. In this Figure, $W=W_{8} / \Sigma$.

The sharp edge of the spectrum of $D_{5}$ in the mean field limit smoothes out for finite $V$. In lattice QCD simulations [4], a roughly Gaussian tail inside the gap with a width that scales as $a / \sqrt{V}$ is 
observed. We will provide an analytical explanation of this result by means of a chiral Lagrangian for the Wilson Dirac spectrum. We will also construct a random matrix theory with the symmetries of the Wilson Dirac operator at $a \neq 0$ that reproduces the zero momentum part of the chiral Lagrangian for the Wilson Dirac operator including $O\left(a^{2}\right)$ terms.

\section{Chiral Lagrangian}

The chiral Lagrangian for Wilson fermions is an expansion in the mass, the momentum and the lattice spacing. Up to a few low-energy constants it is determined uniquely by the transformation properties of the QCD partition function $[8,9,10]$. We decompose the partition function as [3]

$$
Z(m, z ; a)=\sum_{v} Z_{N_{f}}^{v}(m, z ; a)
$$

In the microscopic domain, where $m V, z V$ and $a^{2} V$ are kept fixed for $V \rightarrow \infty$, the partition function with index $v$ is given by (we will see below that $v$ is the index of the Dirac operator)

$$
Z_{N_{f}}^{\chi, v}(m, z ; a)=\int_{U\left(N_{f}\right)} d U \operatorname{det}^{v} U e^{V\left[\frac{1}{2}(m+z) \Sigma \operatorname{Tr} U+\frac{1}{2}(m-z) \Sigma \operatorname{Tr} U^{\dagger}-a^{2} W_{8} \operatorname{Tr}\left(U^{2}+U^{\dagger^{2}}\right)\right]} .
$$

Here, $\Sigma$ is the chiral condensate, $W_{8}$ is a low-energy constant and $z$ is the axial mass. Terms that can be expressed as squares of traces of $U$ and $U^{\dagger}$ are possible [14], but they are suppressed in the the large $N_{c}$-limit [11] and are not considered in this talk. It is instructive to perform a mean field analysis of this chiral Lagrangian valid in the thermodynamic limit at fixed $m$ and $a$. It turns out that the properties of the partition function depend crucially on the sign of $W_{8}$.

$W_{8}>0$ : For $z=0$ the partition function shows a second order phase transition at $m \Sigma=8 a^{2} W_{8}$. At this point the quark mass hits the cloud of eigenvalues of $D_{W}$. The spectrum of $D_{5}$ follows from the $z$-dependence of the partition function. It has a gap $\left[-z_{g}, z_{g}\right]$ that closes at the critical point, where

$$
z_{g}=m\left(1-\left(8 a^{2} W_{8} / m \Sigma\right)^{2 / 3}\right)^{3 / 2} .
$$

$W_{8}<0$ : For $z=0$ the partition function does not undergo a phase transition. This implies that the eigenvalues of $D_{W}$ are not scattered in the complex plane which is consistent with an anti-Hermitian Dirac operator, and violates the very premise we started form. One possibility is that $D_{W}$ would be both anti-Hermitian and $\gamma_{5}$-Hermitian, but then $\left\{\gamma_{5}, D_{W}\right\}=0$, which is not the case.

\subsection{QCD inequalities and the sign of $W_{8}$}

A direct consequence of $D_{5}^{\dagger}=D_{5}$ is the QCD inequality

$$
Z_{N_{f}=2}^{\mathrm{QCD}, v}(m, z)=\left\langle\operatorname{det}^{2}\left(\gamma_{5}\left(D_{W}+m\right)+z\right)\right\rangle>0 \quad \text { for } \quad m, z \quad \text { real. }
$$

For an anti-Hermitian Dirac operator we have that

$$
i^{-2 \operatorname{dim}(D)} Z_{N_{f}=2}^{\mathrm{QCD}, v}(m, z)=i^{-2 \operatorname{dim}(D)}\left\langle\operatorname{det}^{2}\left(\left(D_{W}+i m\right)+i z\right)\right\rangle>0 \quad \text { for } \quad m, z \quad \text { real, }
$$

where $\operatorname{dim}(D)$ is the total dimension of the Dirac matrix. In the continuum limit, $i^{2 \operatorname{dim}(D)}=(-1)^{v}$. 
By changing variables $U \rightarrow i U$ in the chiral partition function (2.2) it follows that

$$
Z_{v}^{\chi, N_{f}}\left(0,0, W_{8}\right)=(i)^{N_{f} v} Z_{v}^{\chi, N_{f}}\left(0,0,-W_{8}\right) .
$$

Since for large mass the partition functions for $+W_{8}$ and $-W_{8}$ have the same sign, one of them must change sign as a function of $m$, and not both signs of $W_{8}$ can be allowed by the QCD inequalities. In Fig. 2 we show the $N_{f}=2$ partition function for $v=1$ as a function of $m$ (left) and as a function of $i m$ (right). The blue curves show the $W_{8}>0$ results, whereas $W_{8}<0$ for the red curves.
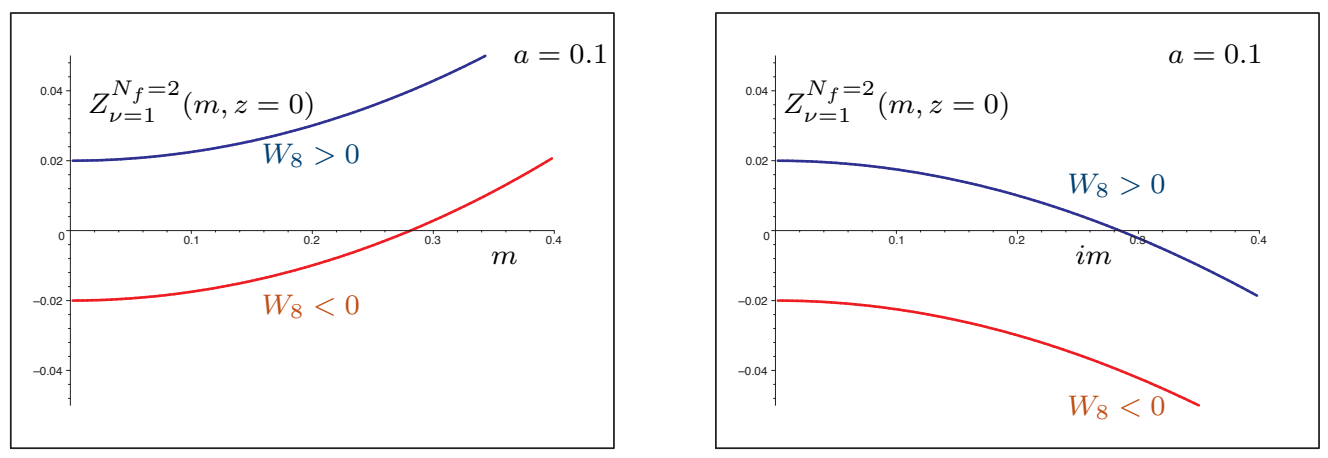

Figure 2: The mass dependence of the partition function (2.2) for real mass (left) and imaginary mass (right).

The conclusion is that $W_{8}>0$ can only correspond to a $\gamma_{5}$-Hermitian Dirac operator, whereas for $W_{8}<0$ the chiral partition function only has a definite sign for imaginary mass so that the corresponding Dirac operator must be anti-Hermitian.

\section{Dirac Spectrum}

To find the Dirac spectrum of $D_{5}$ at $z$ we have to add a fermionic quark with axial mass $z$ and a bosonic quark with mass $z^{\prime}$ to the partition function so that the generating function is given by

$$
Z^{\mathrm{QCD}, v}\left(m, m^{\prime}, z, z^{\prime} ; a\right)=\left\langle\frac{\operatorname{det}\left(\gamma_{5}\left(D_{W}+m\right)+z\right)}{\operatorname{det}\left(\gamma_{5}\left(D_{W}+m^{\prime}\right)+z^{\prime}-i \varepsilon\right)} \prod_{f} \operatorname{det}\left(D_{W}+m_{f}\right)\right\rangle .
$$

The infinitesimal $i \varepsilon$ term is essential. The resolvent

$$
\left.\frac{d}{d z} Z^{\mathrm{QCD}, v}\left(m, m, z, z^{\prime} ; a\right)\right|_{z^{\prime}=z}=\left\langle\operatorname{Tr} \frac{1}{\gamma_{5}\left(D_{W}+m\right)+z-i \varepsilon} \prod_{f} \operatorname{det}\left(D_{W}+m_{f}\right)\right\rangle .
$$

is discontinuous across the support of the spectrum, and for real $z$ its imaginary part gives the spectral density of the Dirac operator (denoted by $\rho_{5}^{v}(z)$ ). The chiral Lagrangian corresponding to the generating function (3.1) is uniquely determined by symmetries. What is not determined by symmetries is the integration contour of the group manifold. For compact integrals this is not an issue, but for noncompact integrals corresponding to bosonic quarks, a change in integration contour may lead to a different analytical continuation of the integral in its complex parameter plane $[12,13,7]$. When $D_{W}$ is Hermitian or anti-Hermitian it is possible to analytically continue 
the partition function in the entire complex $m$-plane. When $D_{W}$ is non-Hermitian the generating function is not analytic if $m$ is inside the support of the spectrum and the expression (3.2) cannot be used to derive the Dirac spectrum.

For a $\gamma_{5}$-Hermitian Dirac operator the partition function (3.1) is an analytical function of $z$, and only the part of the complex $a$-plane to which the partition function can be continued analytically can correspond to lattice QCD at $a \neq 0$. In particular, analyticity in $z$ requires that $\operatorname{Re}\left(a^{2} W_{8}\right)>0$.

The second interesting observable that can be calculated from the generating function is

$$
\Sigma^{v}(m)=\left.\frac{d}{d m} \log Z^{v}\left(m, m^{\prime}, z, z^{\prime} ; a\right)\right|_{z^{\prime}=z=0, m^{\prime}=m} .
$$

For small $a$ it can be shown that the imaginary part of this quantity gives the chirality distribution

$$
\chi^{v}(m)=\left\langle\sum_{k, \lambda_{k}^{W} \in \mathbb{R}} \delta\left(m-\lambda_{k}^{W}\right) \operatorname{sign}\left(\left\langle k\left|\gamma_{5}\right| k\right\rangle\right)\right\rangle,
$$

where $\lambda_{k}^{W}$ are the eigenvalues of $D_{W}$. The integral $\int d m \chi^{v}(m)$ is equal to the index of the Dirac operator. It is defined configuration by configuration as $\sum_{k, \lambda_{k}{ }_{k} \in \mathbb{R}} \operatorname{sign}\left(\left\langle k\left|\gamma_{5}\right| k\right\rangle\right)$.

In the microscopic domain of QCD, the generating function (3.2) is given by an integral over the zero momentum part of the Goldstone manifold

$$
Z_{1 \mid 1}^{\mathrm{SUSY}, v}\left(m, m^{\prime}, z, z^{\prime} ; \hat{a}\right)=\int d U \operatorname{Sdet}(U)^{v} e^{i \frac{1}{2} \operatorname{STr}\left(\mathscr{M}\left[U-U^{-1}\right]\right)+i \frac{1}{2} \operatorname{STr}\left(\mathscr{Z}\left[U+U^{-1}\right]\right)+\hat{a}^{2} \operatorname{STr}\left(U^{2}+U^{-2}\right)}
$$

The integration is over the maximum Riemannian graded submanifold of $G l(1 \mid 1)$ [12] and can be worked out analytically. The result is a sum of products of simple one-dimensional integrals [3]. Calculating the integral $\int d m \chi^{v}(m)$ we find that it is identically equal to $v$. This shows that the parameter $v$ in the chiral Lagrangian is the index of the Dirac operator defined below Eq. (3.4).

It is straightforward to extend this result for QCD with dynamical quarks. The result for $N_{f}=1$ is discussed in [15] in this volume.

\section{Random Matrix Theory}

Since the chiral Lagrangians (2.2) and (3.5) are determined uniquely by the global symmetries of QCD, they can also be obtained from a random matrix theory with the same symmetries. The random matrix partition with index $v$ is defined by [3]

$$
Z_{N_{f}}^{\mathrm{RMT}, v}=\int d A d B d W \operatorname{det}^{N_{f}}\left(D_{W}+m+z \gamma_{5}\right) P\left(D_{W}\right),
$$

with

$$
D_{W}=\left(\begin{array}{cc}
a A & C \\
-C^{\dagger} & a B
\end{array}\right) \quad \text { and } \quad A^{\dagger}=A, \quad B^{\dagger}=B .
$$

Here, $A$ is a square matrix of size $n \times n$, and $B$ is a square matrix of size $(n+v) \times(n+v)$. The matrix $C$ is a complex $n \times(n+v)$ matrix. The matrix elements of $D_{W}$ are taken to be distributed with Gaussian weight

$$
P(A, B, W) \equiv e^{-\frac{N}{4} \operatorname{Tr}\left[A^{2}+B^{2}\right]-\frac{N}{2} \operatorname{Tr}\left[W W^{\dagger}\right]},
$$


where $N=2 n+v$. For $a \rightarrow 0$ the random matrix Dirac operator $D_{W}$ has exactly $v$ zero eigenvalues. For nonzero $a$, the index of the Dirac operator defined below Eq. (3.4) is exactly equal to $v$ for each matrix in the ensemble.

In the microscopic domain, this random matrix theory partition function reduces to the chiral Lagrangian introduced in Eq. (2.2) with $\Sigma=N / V$ and $W_{8}=\frac{1}{2} a^{2} N$. Notice that $W_{8}$ is positive.

\section{Results for the Dirac Spectrum}

Analytical expressions for $\rho_{5}^{v}(x)$ and $\chi^{v}(m)$ that follow from (3.5) were obtained in [3] (see Figs. 3 and 4 for plots). For $a=0$ the eigenvalue density of $D_{5}$ can be decomposed as

$$
\rho_{5}^{v}(x)=v \delta(x-m)+\rho_{x>m}(x) .
$$

For $a \neq 0$ the width of the peak at $x=m$ becomes finite. In Fig. 3 we show the spectral density of $D_{5}$ for $v=1$ and $a=0.05$. Also shown is the $a^{2} W_{8} V \ll 1$ analytical result for the density of the "topological" eigenvalues for $v=1$ which is given by a simple Gaussian

$$
\rho_{5, \text { topo }}^{v=1}(x)=\frac{1}{4 a \sqrt{\pi V W_{8}}} e^{-\frac{V \Sigma^{2}(x-m)^{2}}{16 a^{2} W_{8}}} .
$$

For small $a$, it can be shown for arbitrary $v$ that this distribution approaches the spectral density of the real eigenvalues of $D_{W}$ up to a shift by $m$ [14]. This figure suggests that it is possible to extract the low-energy constant $W_{8}$ from the width of the distribution of the smallest eigenvalue.

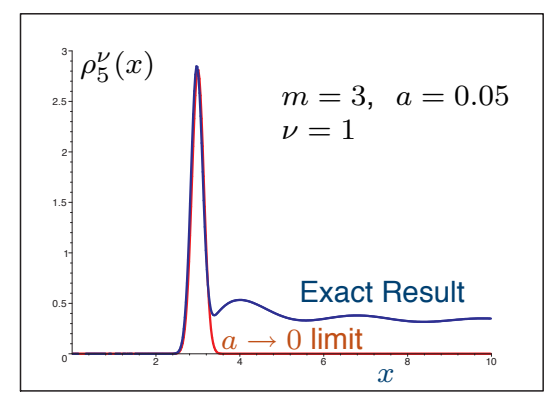

Figure 3: Microscopic spectral density of $\gamma_{5}\left(D_{W}+m\right)$ for $v=1$ (blue curve). Also shown is the distribution of the smallest eigenvalue for $v=1$ obtained in the $a \rightarrow 0$ limit.

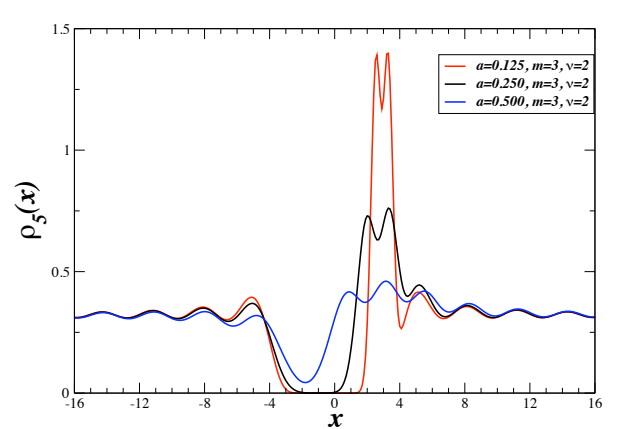

Figure 4: The microscopic spectrum of $\gamma_{5}\left(D_{W}+m\right)$ for $m V \Sigma=3, v=2$ and $a \sqrt{W_{8} V}=$ $0.125,0.250$ and 0.500 , respectively.

For $|x-m| / a$ fixed for $a \rightarrow 0$ the spectral density inside the gap can also be obtained from a saddle point analysis:

$$
\rho_{5}(x) \sim e^{-\Sigma^{2} V(x-m)^{2} / 16 a^{2} W_{8}} \quad \text { for } \quad 0<x \ll m .
$$

The width parameter is given by $\sigma^{2}=8 a^{2} W_{8} / V \Sigma^{2}$, and the ratio of $\sigma$ and and $\Delta \lambda=\pi / \Sigma V$ is equal to $\sigma / \Delta \lambda=\sqrt{8} / \pi a \sqrt{W_{8} V}$ [3]. This is exactly the scaling behavior found in lattice QCD simulations [4]. We note that in this reference the results are an average over different values of $v$. Our results show that, in particular for small $a$, the Wilson Dirac spectrum shows a strong dependence on $v$, and it would be very interesting to analyze the lattice results for fixed index of the Dirac operator. 


\section{Conclusions}

Using chiral perturbation theory we have obtained exact analytical expressions for the spectrum of the Wilson Dirac operator in the microscopic domain. Our result are obtained for fixed value of the index of the Dirac operator and corrections up to $O\left(a^{2}\right)$ have been included. We have formulated a random matrix theory that in the limit of large matrices reproduces the chiral Lagrangian and the analytical results for the Dirac spectrum. Using QCD inequalities for the Wilson Dirac operator we find that the sign of the coefficient of $O\left(a^{2}\right)$ in the chiral Lagrangian is consistent with the existence of an Aoki phase. In the limit of small $a$ we have obtained analytical results for the distribution of the smallest Dirac eigenvalues with fixed nonzero index of the Dirac operator. We find that the width of the distribution scales as $a / \sqrt{V}$ in agreement with earlier lattice simulations. Our results make it possible to extract additional low-energy constants of Wilson chiral perturbation theory from the distribution of the smallest Dirac eigenvalues.

Acknowledgments: We would like to thank many participants of the Lattice 2010 Symposium and the CERN TH-Institute "Future directions in lattice gauge theory - LGT10" for discussions. Two of us (GA and JV) thank the Niels Bohr Foundation for support and the Niels Bohr Institute and the Niels Bohr International Academy for its hospitality. This work was supported by U.S. DOE Grant No. DE-FG-88ER40388 (JV) and the Danish Natural Science Research Council (KS).

\section{References}

[1] E. V. Shuryak and J. J. M. Verbaarschot, Nucl. Phys. A 560, 306 (1993) [hep-th/9212088]; J. J. M. Verbaarschot, Phys. Rev. Lett. 72, 2531 (1994) [hep-th/9401059].

[2] J. Gasser and H. Leutwyler, Phys. Lett. B 184, 83 (1987).

[3] P. H. Damgaard, K. Splittorff and J. J. M. Verbaarschot, Phys. Rev. Lett. 105 (2010) [arXiv:1001.2937].

[4] L. Del Debbio, L. Giusti, M. Luscher, R. Petronzio and N. Tantalo, JHEP 0602, 011 (2006) [hep-lat/0512021]; JHEP 0702, 056 (2007) [hep-lat/0610059].

[5] M. Luscher, JHEP 0707, 081 (2007) [arXiv:0706.2298 [hep-lat]].

[6] S. R. Sharpe, Phys. Rev. D 74,014512 (2006) [hep-lat/0606002].

[7] M. Golterman, S. R. Sharpe and R. L. Singleton, Phys. Rev. D 71, 094503 (2005) [arXiv:hep-lat/0501015].

[8] S. R. Sharpe and R. L. Singleton, Phys. Rev. D 58, 074501 (1998) [hep-lat/9804028].

[9] G. Rupak and N. Shoresh, Phys. Rev. 66, 054503 (2002), [arXiv:hep-lat/0201019].

[10] O. Bar, G. Rupak and N. Shoresh, Phys. Rev. D 70, 034508 (2004), [arXiv:hep-lat/0306021].

[11] R. Kaiser and H. Leutwyler, Eur. Phys. J. C 17, 623 (2000) [hep-ph/0007101].

[12] P. H. Damgaard, J. C. Osborn, D. Toublan and J. J. M. Verbaarschot, Nucl. Phys. B 547, 305 (1999) [hep-th/9811212].

[13] K. Splittorff and J. J. M. Verbaarschot, Nucl. Phys. B 683, 467 (2004) [arXiv:hep-th/0310271].

[14] G. Akemann, P. H. Damgaard, K. Splittorff and J. J. M. Verbaarschot, to be submitted.

[15] G. Akemann, P. H. Damgaard, K. Splittorff and J. J. M. Verbaarschot, this volume. 